\title{
Boundary lubrication effect of organic residue left on surface after evaporation of organic cleaning solvent
}

\author{
Anthony J. Barthel, ${ }^{1}$ Jiawei Luo, ${ }^{1}$ Ki Seob Hwang, ${ }^{2}$ Jun-Young Lee, ${ }^{2}$ and Seong H. Kim ${ }^{1} *$ \\ 1. Department of Chemical Engineering and Materials Research Institute, Pennsylvania \\ State University, University Park, Pennsylvania, 16802 \\ 2. Korea Institute of Industrial Technology, 89 Yangdaegiro-gil, Ipjang-myeon, Seobuk-gu, \\ Cheonan-si, Chungcheongnam-do, 331-822, Korea \\ * Corresponding author: shkim@engr.psu.edu
}

\begin{abstract}
Although samples are visibly clean, organic deposits could have tremendous impacts on tribological measurements and interpretation of friction and wear behaviors. This paper discusses the boundary lubrication effects of invisible residues from organic solvents that have been widely used in tribological studies in ambient conditions. Stainless steel, soda lime glass, and copper substrates were cleaned using UV/ozone treatment or organic solvents such as alcohols where the solvent was allowed to evaporate from the surface. In ball-on-flat tribo-tests, all UV/ozone cleaned samples showed high friction and catastrophic wear immediately upon sliding due to the absence of lubricants at the sliding interface, while samples cleaned by organic solvent experienced low friction and minimal wear for hundreds of reciprocating cycles. Analyses of the surface by atomic force microscopy and vibrational spectroscopy indicated that the solvent cleaning deposits hydrocarbon residue on metal and oxide substrates. This residue is due to trace impurities with low vapor pressures inevitably present in the bulk liquid, regardless of solvent


purity, which become concentrated upon vaporization of the high vapor pressure solvent. These results demonstrated that drying after cleaning with organic solvents is equivalent to a dipcoating of low vapor pressure organics onto the sample surface. This dip-coating effect can be avoided if surfaces are cleaned using UV/ozone or if the surface is immediately rinsed with DI water and blow-dried after cleaning with organic solvent. These results indicate that the friction coefficients of the samples with organic residues should not be interpreted with the shear strength model of friction. 


\section{Introduction}

Sample cleanliness is crucial for accurate measurements of friction and wear behaviors of sliding surfaces. Dry sliding between two surfaces that are in intimate contact can be affected by a very thin layer of lubricant. Self assembled monolayers (SAMs) have been shown to lubricate surfaces in atomic force microscopy (AFM) studies as well as decrease adhesion in microelectromechanical systems (MEMS) [1,2]. Many solid surfaces show a low friction coefficient near 0.2 and minimal wear with only a monolayer of adsorbed alcohol vapor [3,4]. This lubrication occurs not only at the nano-scale; it is also observed at the macro-scale where the surface roughness is several orders of magnitude larger than the size of adsorbed molecules [3]. Vapor can also be used to deliver a thin molecular coating to protect surfaces from wear [57].

Crucial to the proper function of these boundary lubrication methods is careful control of the chemical species on the surface. In the absence of any coating, pure metal surfaces could irreversibly adhere to one another and friction coefficients for these materials could be much higher than one [8-10]. Exposure to ambient air will form oxide layers on substrates with high surface energy [11-13]. It can also introduce a thin layer of organic contaminants adsorbed from the gas phase. These organic molecules can react to form adventitious carbonaceous deposits on surfaces in assistance of frictional heat or electricity, which can influence the tribological properties of the system [14-16].

This paper examines the effect of cleaning tribological surfaces with commonly-used organic solvents, with specific focus on the residue left after solvent evaporation. Soda lime glass (SLG), stainless steel (SS), and copper substrates were cleaned using two methods: (i) 
UV/ozone and (ii) rinsing with alcohol followed by evaporation or blow dry from the sample surface. The experimental results indicated that the residue film resulting from the evaporated liquid lubricates SLG, SS, and Cu surfaces with low friction and virtually no wear for a certain period which varies depending on the residue film thickness and sliding conditions. Since SLG, SS, and $\mathrm{Cu}$ have very different surface chemistry and physical properties, this effect is not a material specific phenomenon. Even if only a trace amount of residues is present in ultra-high purity solvent, the evaporation of solvent results in concentration of these residues on the substrate. Thus, the solvent cleaning and evaporation could be considered or equivalent to a dipcoating of low vapor pressure organic residues which inevitably exist in liquid solvents. Based on the findings from this study, the shear strength model of friction for unlubricated solids is revisited $[17,18]$.

\section{Experimental}

Friction tests were conducted using a custom-built reciprocating ball-on-flat tribometer [19]. Substrate material was AISI 440C stainless steel (SS), oxygen-free high-conductivity (OFHC) copper $(\mathrm{Cu})$, and soda lime slide glass (SLG). Commercially available $3 \mathrm{~mm}$ diameter 440C stainless steel balls were used as a counter-surface for all copper and stainless steel substrate tests; $2.4 \mathrm{~mm}$ diameter borosilicate glass balls were used for SLG substrates. The tribometer was operated at a sliding speed of $4 \mathrm{~mm} / \mathrm{s}$ with a $1 \mathrm{~N}$ load for each test and slide tracks were roughly $2.5 \mathrm{~mm}$ long. All tests were conducted in a gas environment of dry nitrogen and were repeated for at least 5 times. The nominal Hertzian contact pressure for SS ball against SS substrate, SS ball against copper substrate, and glass balls against SLG substrate are calculated to be $642 \mathrm{MPa}, 524 \mathrm{MPa}$, and $350 \mathrm{MPa}$ respectively. Previous vapor phase lubrication studies 
showed that SS, Cu, and SLG substrates do not get damaged mechanically as long as the surface is covered with one monolayer of alcohol molecules $[3,12]$. Thus, friction and wear tests at these contact conditions are sensitive to chemical conditions of the sliding interface.

All substrate samples were cleaned by rinsing with ethanol, followed by exposure to UV/ozone cleaning for at least 20 minutes. Tribotests investigating this surface were conducted immediately following UV exposure. For tests investigating the effect of cleaning with liquid solvent, the sample was taken from the UV/ozone chamber and then rinsed by the liquid in question, with liquid left on the sample to evaporate in air. Liquids investigated were ethanol, methanol, isopropanol, and decane, and each liquid had purity greater than 99\%. After drying, these samples were tested using the tribometer.

Infrared spectroscopy analysis was carried out using a Thermo-Nicolet Nexus 670 system equipped with a MCT detector. Attenuated total reflection (ATR) tests were conducted for a liquid sample using a multi-bounce silicon crystal [20]. Polarization-modulation reflectionabsorption infrared spectroscopy (PM-RAIRS) was used to analyze organic species remaining on metal surfaces; detailed description of the setup is available elsewhere [19]. For chemical analysis of organic species on SLG surfaces, vibrational sum frequency generation (SFG) spectroscopy was used [21]. Briefly, the SFG signal was generated at the substrate/air interface where two laser pulses $-532 \mathrm{~nm}$ visible pulses with $60^{\circ}$ incident angle from an EKSPLA Nd:YAG laser and tunable IR pulses with $56^{\circ}$ incident angle generated with an optical parameter generator and amplifier - were spatially and temporally overlapped. The SFG signal was collected in a reflection geometry and normalized with the input intensities of visible and IR pulses. The polarization of light was $s$ for SFG signal, $s$ for visible beam, and $p$ for IR beam (ssp). 

in ambient environment. The surface topography of samples with different cleaning methods were investigated by using atomic force microscopy (AFM) with a Digital Instrument MultiMode scanning probe microscope. Topographic images of the sample surface were collected with a contact-mode scanning. The AFM image size was $5 \mu \mathrm{m} \times 5 \mu \mathrm{m}$.

\section{Results and Discussion}

The lubrication of evaporated solvent residue was tested on glass, stainless steel, and copper. Figure 1 compares the friction behavior for stainless steel (SS), soda lime glass (SLG), and copper cleaned by either UV-ozone or by liquid ethanol. All tests were conducted in dry nitrogen. Figure 2 shows the surface profile of each material after friction tests. The severely worn surfaces with wear depth deeper than several microns (Figures 2a, 2c, and 2e) were observed for the samples cleaned with UV/ozone. When sliding was stopped before friction increased abruptly, the ethanol-cleaned surfaces showed only plastic deformation and no significant wear (Figures 2b, 2d, and 2f). 


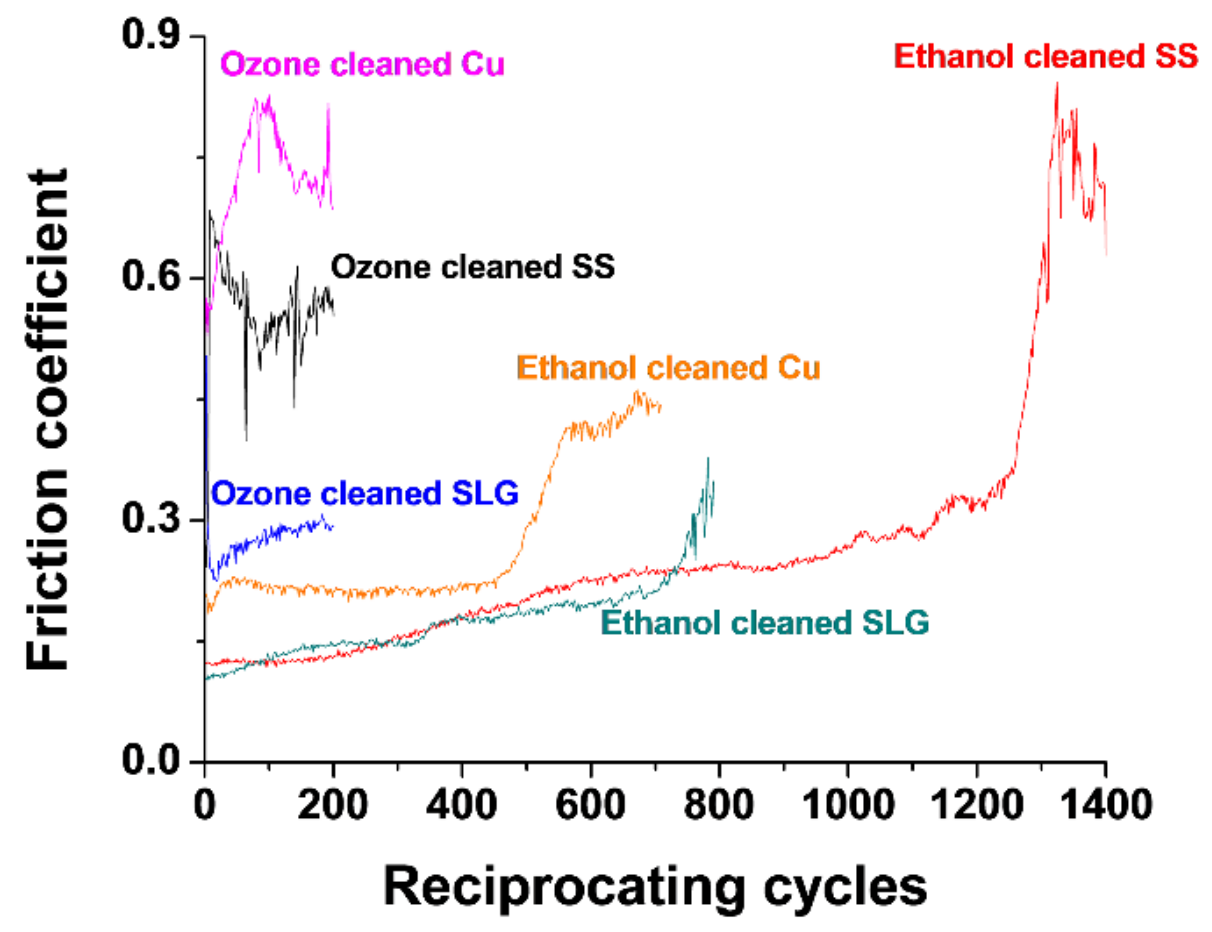

Figure 1: Friction coefficient for various substrates cleaned by UV/ozone or liquid ethanol. All tests conducted in dry nitrogen environment. 

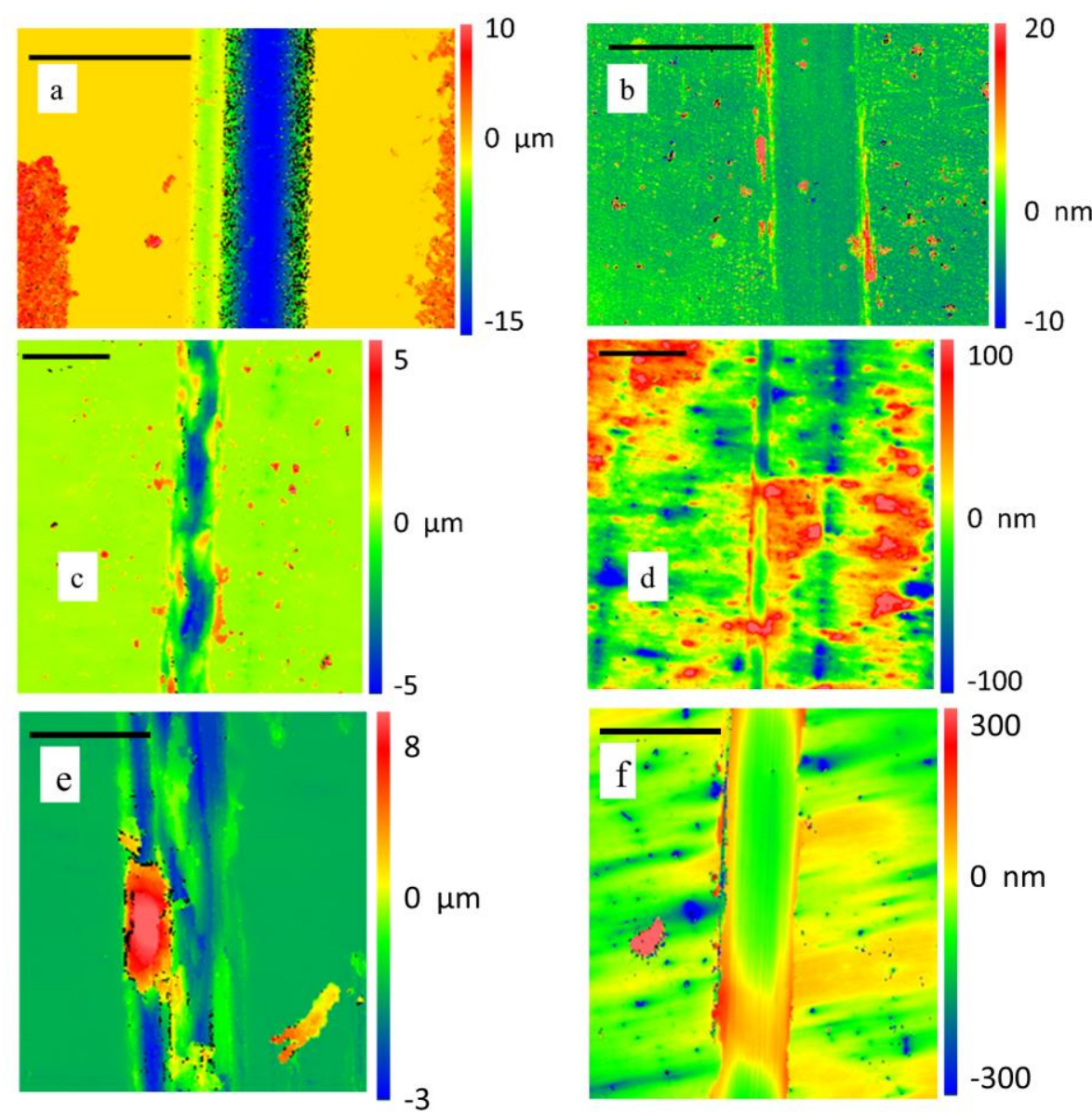

Figure 2: Optical profilometry images for SLG, SS, and Cu wear tracks. SLG is depicted in "a" (ozone cleaned) and "b" (ethanol cleaned); SS is depicted in "c" (ozone cleaned) and "d" (ethanol cleaned) and $\mathrm{Cu}$ is depicted in "e" (ozone cleaned) and " $\mathrm{f}$ " (ethanol cleaned). All scale bars are $100 \mu \mathrm{m}$ and the color legend is to the right of each image. Polishing scratches for SS and $\mathrm{Cu}$ are horizontal.

Both friction and wear behaviors show that there is a drastic difference between the surface cleaned by UV-ozone and the surface cleaned by ethanol. All materials cleaned by UVozone experience wear and high friction immediately at the start of the tribotest. The value near 
0.3 is typical of SLG substrate wear, which occurs through scratching/abrasion and creates piles of particulate debris, which is evident at the far edges of the optical profilometry image in Figure 2a. The friction value for SS is higher than 0.5 and the surface is severely damaged due to adhesive wear, as seen in Figure 2c. Similar behavior is seen for $\mathrm{Cu}$ in Figure 2e, with a friction coefficient higher than 0.6. The worn tracks from the UV-ozone cleaned surface measure several microns deep for each material.

These friction and wear patterns are in contrast to the negligible wear and low friction observed for the ethanol-cleaned samples. Friction for SS and SLG begins near 0.1 and slowly rises towards 0.2 as sliding continues, while friction for $\mathrm{Cu}$ is constant near 0.18 until wear occurs. Wear, or evidence of material removal by rubbing, is nearly imperceptible on the materials during the low friction period. The sliding track on SLG (Figure 2b) shows no height difference from the surrounding surface and is discernible mainly due to the minute pile-up of material at the periphery of the sliding track, whose height measures only tens of nanometers. The SS sliding track is virtually wearless (Figure 2d); only a slight depression due to plastic deformation in the bulk can be recognized. The $\mathrm{Cu}$ track (Figure $2 \mathrm{f}$ ) is somewhat easier to see due to larger plastic deformation of the softer copper substrate.

The residual deposits from ethanol cleaning were further investigated by AFM. Figure 3 shows topographic images for a glass surface cleaned by liquid ethanol and then the same surfaces after cleaning with UV/ozone. The images from the ethanol-cleaned surface clearly show residual deposits distributed around the sample that measure up to $4 \mathrm{~nm}$ (Figure $3 \mathrm{a}$ ). The glass surface becomes essentially featureless in the topographic image after cleaning with UV/ozone as seen in Figure $3 b$. 

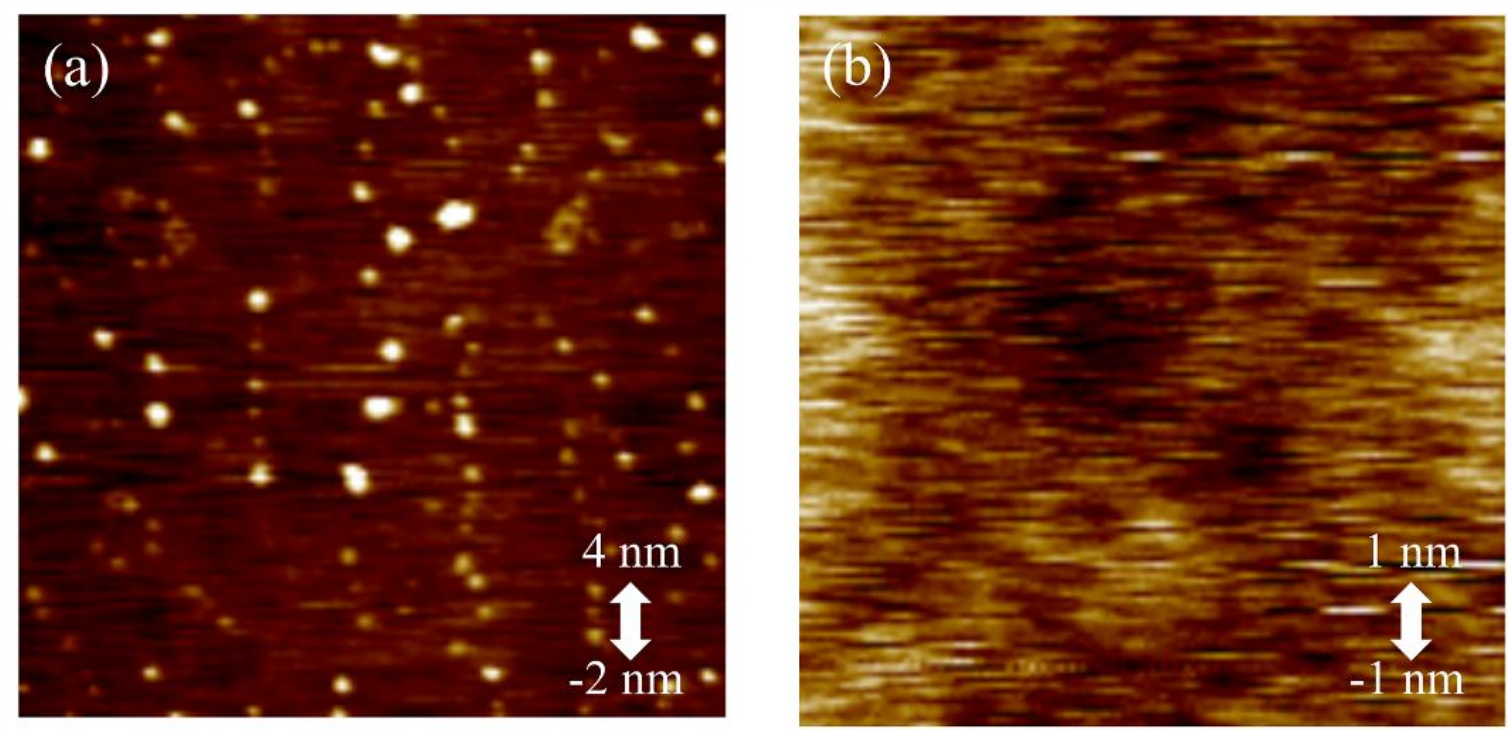

Figure 3: AFM topography image for cleaned glass surfaces: (a) Ethanol-cleaned SLG surface;

(b) Ethanol-cleaned and then UV-ozone cleaned SLG surface. Brighter color represents a higher value and darker color represents a lower value. The size of the images is $5 \mu \mathrm{m} \times 5 \mu \mathrm{m}$.

The chemical nature of the organic material left behind by ethanol cleaning was investigated by PM-RAIRS and SFG. Figure 4 displays the spectral difference between liquid ethanol taken by ATR-IR and the deposit from evaporated liquid ethanol on copper taken with PM-RAIRS. Only the spectrum of the ethanol-cleaned copper substrate is shown here because copper gives better PM-RAIRS spectra due to its better IR reflectivity than SS and SLG, but the nature of remaining chemical species is expected to be the same for SS and SLG [22]. The evaporated ethanol spectrum shows a sharp peak near $2925 \mathrm{~cm}^{-1}$ and another near $2850 \mathrm{~cm}^{-1}$, typical of $\mathrm{C}-\mathrm{H}$ stretching of $\mathrm{CH}_{2}$ groups. Note that these peaks look very similar to the $\mathrm{CH}_{2}$ asymmetric and symmetric stretch modes, respectively, of long-chain alkyl groups as seen in various SAMs and alkane molecules adsorbed on metal surfaces [3,23-25]. These alkyl stretching vibrations are different than those seen in liquid ethanol. The strongest peaks in liquid 
ethanol occur at $2975 \mathrm{~cm}^{-1}$ and $2890 \mathrm{~cm}^{-1}$, with a shoulder near $2930 \mathrm{~cm}^{-1}$. These peaks represent the $\mathrm{CH}_{3}$ asymmetric stretch $\left(2975 \mathrm{~cm}^{-1}\right)$, the $\mathrm{CH}_{2}$ Fermi resonance or $\mathrm{CH}_{2}$ asymmetric stretch $\left(2930 \mathrm{~cm}^{-1}\right)$, and the $\mathrm{CH}_{3}$ symmetric stretch $\left(2890 \mathrm{~cm}^{-1}\right)$ [26,27]. Absent from the evaporated liquid spectrum is the $\mathrm{OH}$ signal of alcohol centered near $3330 \mathrm{~cm}^{-1}$, which is very strong and broad in the liquid spectrum. The absence of the $\mathrm{OH}$ stretching peak as well as the peak positions of the $\mathrm{C}-\mathrm{H}$ stretching modes indicate that the residue on the copper surface is long-chain hydrocarbons in nature and lacks a hydroxyl group.

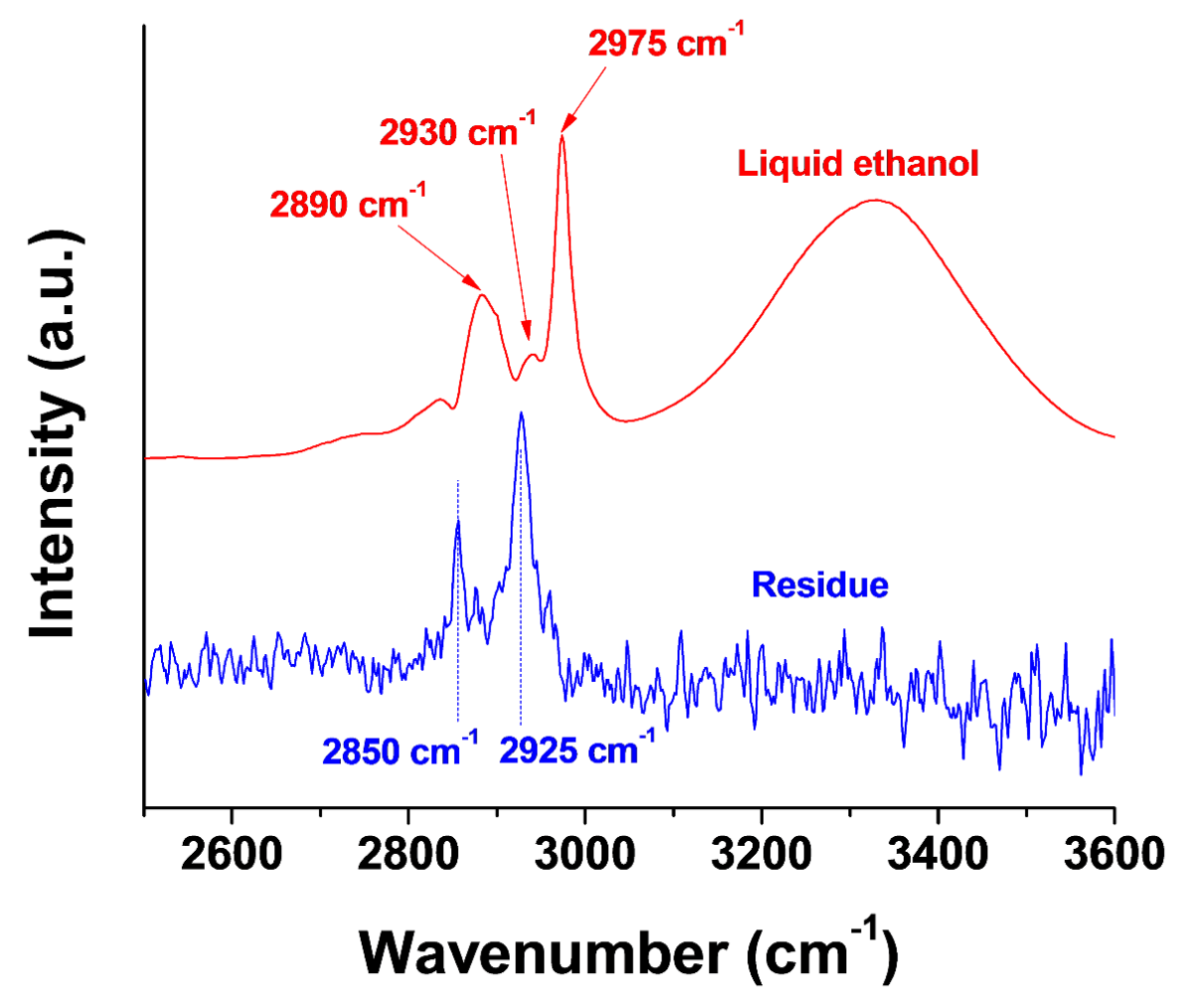

Figure 4: ATR-IR spectrum of liquid ethanol (red) on a Si crystal and PM-RAIRS spectrum of residue from ethanol after evaporation of the liquid (blue) on a $\mathrm{Cu}$ substrate. 
This nature was further investigated with SFG. Figure 5 compares the SFG spectra of SLG slides cleaned in various ways in different gas environments as well as the SFG spectra of liquid-air interface for ethanol and methanol. The liquid ethanol spectrum shows two peaks at $2875 \mathrm{~cm}^{-1}$ and $2930 \mathrm{~cm}^{-1}$. In contrast, both ethanol-cleaned and UV/ozone cleaned SLG surfaces shows three peaks at $2850 \mathrm{~cm}^{-1}, 2875 \mathrm{~cm}^{-1}$, and $2930-2935 \mathrm{~cm}^{-1}$. In $\mathrm{SFG}$, the $2850 \mathrm{~cm}^{-1}$ peak is characteristic to hydrocarbons longer than the ethyl group [28]. Thus, its presence is an evidence for the presence of adventitious hydrocarbon contamination on the UV/ozone cleaned surface exposed to the ambient air.

The SFG intensities of the hydrocarbon peaks are stronger for the ethanol-cleaned surface than the UV/ozone cleaned surface. In addition, there are some differences in spectral features compared to the adventitious hydrocarbon peaks on the UV/ozone cleaned surface. The asymmetric stretch peak is slightly shifted to $2935 \mathrm{~cm}^{-1}$ and the relative intensities of the symmetric stretch peaks $\left(2850 \mathrm{~cm}^{-1}\right.$ and $\left.2875 \mathrm{~cm}^{-1}\right)$ are stronger than the asymmetric peak. Thus, the organic species on the ethanol-cleaned surface appear to be more abundant and different than those on the UV/ozone cleaned surface. 


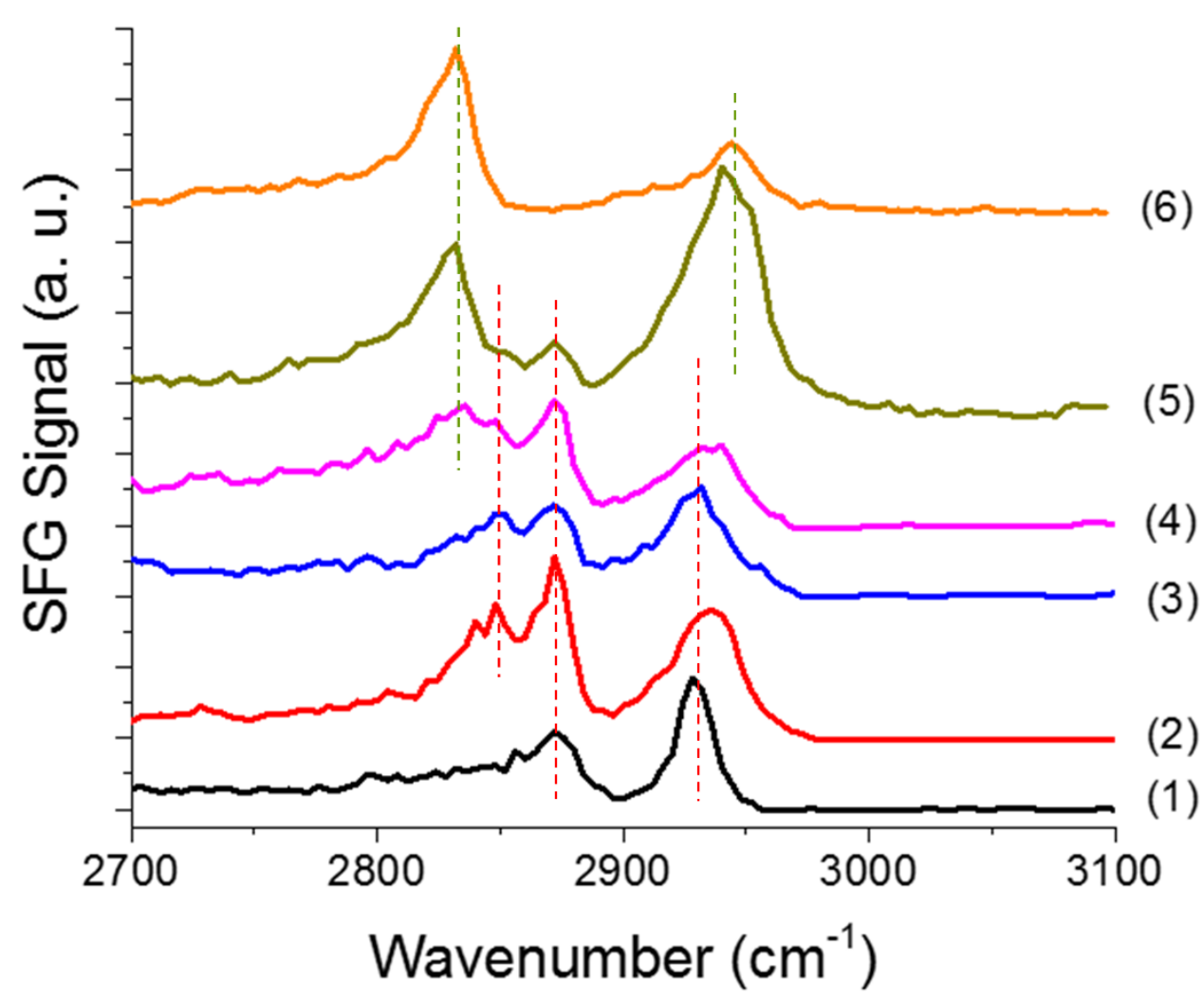

Figure 5: SFG spectra with ssp polarization for (1) liquid ethanol in ambient air, (2) ethanolcleaned soda-lime glass in ambient air, (3) UV/ozone cleaned soda-lime glass in ambient air, (4) ethanol-cleaned soda-lime glass in methanol vapor environment, (5) UV/ozone cleaned sodalime glass in methanol vapor environment, and (6) liquid methanol.

In order to test how these surfaces cleaned with two different methods respond to the exposure to organic vapors, SFG analysis in methanol vapor was carried out. The liquid methanol spectrum has a unique sharp peak near $2830 \mathrm{~cm}^{-1}$, along with a weaker peak near 2940 $\mathrm{cm}^{-1}$ in the $s s p$ SFG spectrum (Figure 5). Since only methoxy group shows the peak at $2830 \mathrm{~cm}^{-}$ ${ }^{1}$ [28], this peak is a good indicator that can be used to distinguish the presence of methanol molecules from other hydrocarbons. Upon exposure to the methanol vapor of the UV/ozone 
cleaned surface, the $2830 \mathrm{~cm}^{-1}$ and $2940 \mathrm{~cm}^{-1}$ peaks of the adsorbed methanol become dominant and much stronger than the adventitious hydrocarbon peaks. This indicates that the UV/ozone cleaned surface is sufficiently clean to allow adsorption of methanol molecules from the vapor phase. The relative intensities of two peaks of the adsorbed methanol are different from those of the liquid methanol. This must be due to the orientation difference of methanol molecules at the solid and liquid surfaces [28]. This result is consistent with the fact that the UV/ozone cleaned surfaces exhibit high friction in dry nitrogen and strong dependences on chemical composition of the surrounding gas environment $[3,4,29]$.

In the case of the ethanol-cleaned surface, the growth of $2830 \mathrm{~cm}^{-1}$ peak is also noticeable upon exposure to the methanol vapor, but not as prominent as the UV/ozone cleaned surface. Moreover, the asymmetric stretch peak is still centered at $2930 \mathrm{~cm}^{-1}$, instead of $2940 \mathrm{~cm}^{-1}$. This result implies that the methanol adsorption occurs, but its surface coverage is very small or limited since the surface is already covered with organic deposits from the ethanol solvent. Thus, the friction and wear behavior of the ethanol-cleaned surface would be dominant by the organic deposits from the solvent, rather than the molecules adsorbed from the gas phase.

The low friction behavior that results from evaporated ethanol is also seen for other organic liquids. Figure 6 compares friction behaviors for SLG that was cleaned with UV/ozone versus different liquids. These liquids include an alkane, a branched alcohol (isopropanol which is another commonly used cleaning solvent) as well as the simplest alcohol (methanol). The liquid-cleaned surfaces exhibit low and stable friction near 0.1 or below for the duration of the experiment and the corresponding glass surface (not shown) shows no wear. Differences in friction coefficient may be due to different thickness or types of residue film [30]. 


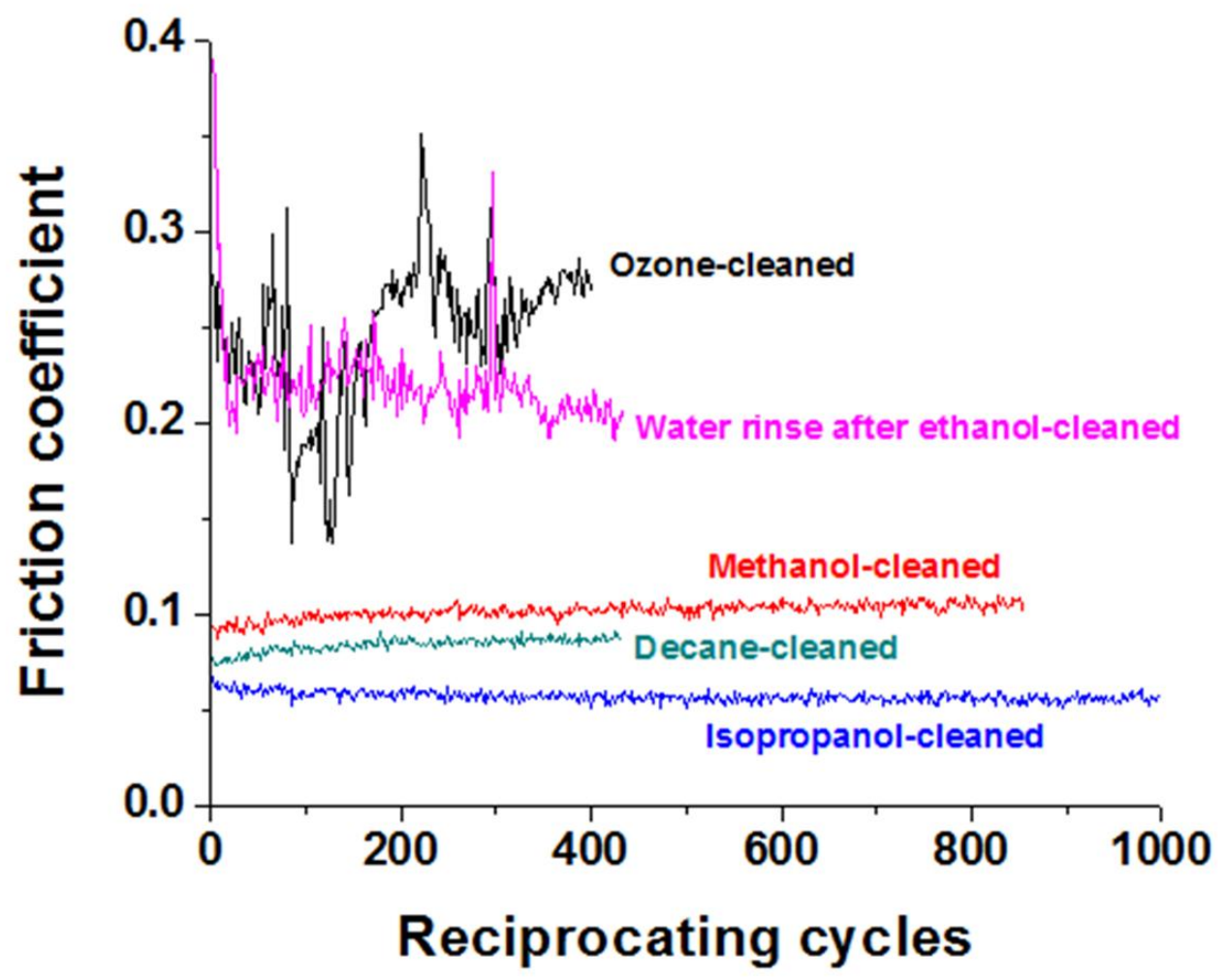

Figure 6: Friction coefficient for the resulting residue from different evaporated solvents compared to a UV/ozone cleaned surface. All data comes from SLG substrates and tests were conducted in dry $\mathrm{N}_{2}$.

Figure 6 also includes results from a sample that was cleaned by liquid ethanol but then rinsed by DI water immediately after ethanol cleaning without allowing evaporation of ethanol. This replaces the organic solvent and residue on the surface with clean water. The water on the sample was then blown away from the testing area. Since water molecules have stronger interactions (for example, hydrogen bonding interactions) with the surface than organics [20], the strongly bound water appears to prevent or minimize the deposition of organic residue on the surface. This resulted in a high friction coefficient similar to the ozone-cleaned surface as well 
as significant ball and substrate wear, indicating that the glass surface is free from organic residual films.

The organic liquids analyzed in Figures 1 to 6 are chemically different from one another, but all share two important characteristics: relatively low surface tension and high vapor pressure. These characteristics help to explain how the lubricating residue is accumulated at the surface. All liquids used were high purity (99\% or higher, depending on chemical). No matter how small impurities are, they can be concentrated upon evaporation of the bulk liquid if the impurity has a vapor pressure lower than that of main solvent. In other words, the evaporation of the solvent ends up with concentrating impurities or additives with low vapor pressure which exist as a trace amount in the solvent. Thus, it will eventually result in a thin film or deposit on solid surfaces after drying. Unless the surface energy (or surface tension) of the solid is lower than that of these impurities, the evaporation process becomes equivalent to the 'dip coating' process [31]. This contaminant can accumulate on the substrate surface as the bulk liquid evaporates, sometimes leaving an invisible film on the surface. This film is composed of the impurities in the liquid as well as any initial contaminant present on the surface.

The value of the friction coefficient for different evaporated liquids appears to be different in Figures 1 and 6, but this cannot be attributed solely to chemical differences between the liquids. The drying liquid may not evenly distribute the resulting film across the substrate, leaving different coverages on the surface by a process known as the coffee-ring effect [32]. Additionally, if the bulk of the film originates from impurities in the liquid, then the amount of liquid that evaporates will dictate the thickness of film formed. A larger amount of liquid over a smaller surface area will result in a thicker film that would give lubrication effects for a longer time. This can also affect the duration of lubrication; the friction coefficient for evaporated 
ethanol in Figure 1 slowly increases on SS and SLG surfaces, while the friction coefficient for the evaporated liquids in Figure 6 is relatively constant. The lubrication from the residue films in Figure 6 will eventually fail and the surface will begin to wear. The uncontrolled nature of residual film deposited on the surface makes quantification or precise chemical determination challenging, but Figures 4 and 5 indicate that the film is fundamentally different from the liquid alcohol.

The value of the friction coefficient of $0.1 \sim 0.2$ falls in the range that has been historically reported for the sliding of "unlubricated" smooth surfaces [17]. This friction coefficient value has been interpreted in a theoretical model considering the tensile strength and hardness of a material for asperity cold-welded junctions in metals. Starting with Bowden and Tabor's theory for real contact area, Popov derived the shear strength necessary to break coldwelded junctions during the slide and arrived at a friction coefficient value of $0.16-0.2$ for nonlubricated pairings of various solid materials [18]. However, it appears to be coincidental that this friction value matches those seen in Figures 1 and 6 for the samples cleaned with organic solvents. The analysis using tensile strength and hardness of the material relies on plastic deformation of the material and local cold-welding of asperities. This may be valid assumptions for most metals, but it cannot be applied to a brittle material like soda-lime glass [33]. The fact that both brittle (SLG) and tensile ( $\mathrm{SS}$ and $\mathrm{Cu}$ ) substrates have virtually the same friction coefficient (Figure 1) indicates that the lubrication due to the residue film is not specific to the material properties, but only requires a flat surface with an invisible film of organic residues. 


\section{Conclusion}

The tribological influence of cleaning by liquid solvents and their subsequent residue deposited by evaporation were investigated. Substrates on which alkane or alcohol solvents were allowed to evaporate showed good boundary lubrication and wear prevention compared to surfaces cleaned by UV/ozone. This lubrication was observed in a dry inert environment which otherwise should have caused high friction and considerable wear. The residue seems to originate from impurities in the liquid which accumulate on the surface as the higher vapor pressure solvent evaporates. This residual film can be negated by cleaning surfaces by UV/ozone or by rinsing a surface with DI water immediately following initial cleaning by organic liquid (before the organic liquid dries out). The theoretical model that attributes the friction coefficient of 0.1-0.2 for unlubricated solid surfaces to the shear strength of materials cannot be applied to the solid surfaces covered with invisible organic residues from cleaning solvents.

Acknowledgements. This work was supported by the National Science Foundation (Grant No.

DMR-1207328 and CMMI-1435766). The SFG work was carried out with the DMR support and the tribological tests and PM-RAIRS analysis were carried out with the CMMI support. KSH and JYL were supported by the Korea Institute of Industrial Technology.

\section{References}

[1] M.J. Brukman, G.O. Marco, T.D. Dunbar, L.D. Boardman, R.W. Carpick, Nanotribological properties of alkanephosphonic acid self-assembled monolayers on aluminum oxide: Effects of fluorination and substrate crystallinity, Langmuir. 22 (2006) 3988-3998. doi:10.1021/la052847k.

[2] R. Maboudian, W.R. Ashurst, C. Carraro, Self-assembled monolayers as anti-stiction coatings for MEMS: characteristics and recent developments, Sensors Actuators A Phys. 82 (2000) 219-223. doi:10.1016/S0924-4247(99)00337-4. 
[3] A.J. Barthel, S.H. Kim, Lubrication by Physisorbed Molecules in Equilibrium with Vapor at Ambient Condition: Effects of Molecular Structure and Substrate Chemistry, Langmuir. 30 (2014) 6469-6478. doi:10.1021/la501049z.

[4] A.L. Barnette, D.B. Asay, D. Kim, B.D. Guyer, H. Lim, M.J. Janik, et al., Experimental and density functional theory study of the tribochemical wear behavior of $\mathrm{SiO} 2$ in humid and alcohol vapor environments, Langmuir. 25 (2009) 13052-13061. doi:10.1021/la901919z.

[5] M.G. Hankins, P.J. Resnick, P.J. Clews, T.M. Mayer, D.R. Wheeler, D.M. Tanner, et al., Vapor deposition of amino-functionalized self-assembled monolayers on MEMS, in: R. Ramesham, D.M. Tanner (Eds.), Proc. SPIE, 2003: pp. 238-247. doi:10.1117/12.478206.

[6] Y.X. Zhuang, O. Hansen, T. Knieling, C. Wang, P. Rombach, W. Lang, et al., Vapor-phase selfassembled monolayers for anti-stiction applications in MEMS, J. Microelectromechanical Syst. 16 (2007) 1451-1460. doi:10.1109/JMEMS.2007.904342.

[7] K.R. Coffey, V. Raman, N. Staud, D.J. Pocker, Vapor lubrication of thin film disks, IEEE Trans. Magn. 30 (1994) 4146-4148. doi:10.1109/20.334017.

[8] D.H. Buckley, Influence of chemisorbed films of various gases on adhesion and friction of tungsten, J. Appl. Phys. 39 (1968) 4224-4233. doi:10.1063/1.1656952.

[9] H. Lee, R. a Coutu, S. Mall, K.D. Leedy, Characterization of metal and metal alloy films as contact materials in MEMS switches, J. Micromechanics Microengineering. 16 (2006) 557-563. doi:10.1088/0960-1317/16/3/011.

[10] C.. McFadden, a. . Gellman, Metallic friction: the effect of molecular adsorbates, Surf. Sci. 409 (1998) 171-182. doi:10.1016/S0039-6028(98)00178-2.

[11] C.T. Campbell, Transition metal oxides: extra thermodynamic stability as thin films., Phys. Rev. Lett. 96 (2006) 066106. doi:10.1103/PhysRevLett.96.066106.

[12] A. Alazizi, A.J. Barthel, N.D. Surdyka, J. Luo, S.H. Kim, Vapors in the ambient-A complication in tribological studies or an engineering solution of tribological problems?, Friction. 3 (2015) 85114. doi:10.1007/s40544-015-0083-5.

[13] A.J. Barthel, A. Al-Azizi, N.D. Surdyka, S.H. Kim, Effects of Gas or Vapor Adsorption on Adhesion, Friction, and Wear of Solid Interfaces, Langmuir. 30 (2014) 2977-2992. doi:10.1021/la402856j.

[14] H.W. Hermance, T.F. Egan, Organic Deposits on Precious Metal Contacts, Bell Syst. Tech. J. 37 (1958) 739-776. doi:10.1002/j.1538-7305.1958.tb03885.x.

[15] L. Chen, H. Lee, Z.J. Guo, N.E. McGruer, K.W. Gilbert, S. Mall, et al., Contact resistance study of noble metals and alloy films using a scanning probe microscope test station, J. Appl. Phys. 102 (2007) 074910. doi:10.1063/1.2785951.

[16] M. Kalin, R. Simič, Atomic force microscopy and tribology study of the adsorption of alcohols on diamond-like carbon coatings and steel, Appl. Surf. Sci. 271 (2013) 317-328. doi:10.1016/j.apsusc.2013.01.192.

[17] N.P. Suh, H.-C. Sin, The genesis of friction, Wear. 69 (1981) 91-114. doi:10.1016/00431648(81)90315-X.

[18] V.L. Popov, Contact Mechanics and Friction, Springer Berlin Heidelberg, Berlin, Heidelberg, 2010. doi:10.1007/978-3-642-10803-7.

[19] A.J. Barthel, M.D. Gregory, S.H. Kim, Humidity effects on friction and wear between dissimilar metals, Tribol. Lett. 48 (2012) 305-313. doi:10.1007/s11249-012-0026-5.

[20] D.B. Asay, S.H. Kim, Evolution of the adsorbed water layer structure on silicon oxide at room 
temperature., J. Phys. Chem. B. 109 (2005) 16760-16763. doi:10.1021/jp053042o.

[21] A.L. Barnette, L.C. Bradley, B.D. Veres, E.P. Schreiner, Y.B. Park, J. Park, et al., Selective detection of crystalline cellulose in plant cell walls with sum-frequency-generation (SFG) vibration spectroscopy, Biomacromolecules. 12 (2011) 2434-2439. doi:10.1021/bm200518n.

[22] R.G. Greenler, Reflection Method for Obtaining the Infrared Spectrum of a Thin Layer on a Metal Surface, J. Chem. Phys. 50 (1969) 1963. doi:10.1063/1.1671315.

[23] A. Tu, H.R. Kwag, A.L. Barnette, S.H. Kim, Water adsorption isotherms on CH3-, OH-, and COOH-terminated organic surfaces at ambient conditions measured with PM-RAIRS, Langmuir. 28 (2012) 15263-15269. doi:10.1021/la302848k.

[24] S. Lee, Y.S. Shon, R. Colorado, R.L. Guenard, T.R. Lee, S.S. Perry, Influence of packing densities and surface order on the frictional properties of alkanethiol self-assembled monolayers (SAMs) on gold: A comparison of SAMs derived from normal and spiroalkanedithiols, Langmuir. 16 (2000) 2220-2224. doi:10.1021/la9909345.

[25] M. Salmeron, Generation of defects in model lubricant monolayers and their contribution to energy dissipation in friction, Tribol. Lett. 10 (2001) 69-79. doi:10.1023/A:1009026312732.

[26] A.L. Barnette, D.B. Asay, M.J. Janik, S.H. Kim, Adsorption Isotherm and Orientation of Alcohols on Hydrophilic SiO 2 under Ambient Conditions, J. Phys. Chem. C. 113 (2009) 10632-10641. doi:10.1021/jp901064r.

[27] H.-F. Wang, W. Gan, R. Lu, Y. Rao, B.-H. Wu, Quantitative spectral and orientational analysis in surface sum frequency generation vibrational spectroscopy (SFG-VS), Int. Rev. Phys. Chem. 24 (2005) 191-256. doi:10.1080/01442350500225894.

[28] R. Lu, W. Gan, B.H. Wu, Z. Zhang, Y. Guo, H.F. Wang, C-H stretching vibrations of methyl, methylene and methine groups at the vapor/Alcohol $(n=1-8)$ interfaces, J. Phys. Chem. B. 109 (2005) 14118-14129. doi:10.1021/jp051565q.

[29] H. He, L. Qian, C.G. Pantano, S.H. Kim, Mechanochemical Wear of Soda Lime Silica Glass in Humid Environments, J. Am. Ceram. Soc. 97 (2014) 2061-2068. doi:10.1111/jace.13014.

[30] K. Kato, Wear in relation to friction - a review, Wear. 241 (2000) 151-157. doi:10.1016/S00431648(00)00382-3.

[31] L.E. Scriven, Physics and Applications of DIP Coating and Spin Coating, MRS Proc. 121 (1988) 717. doi:10.1557/PROC-121-717.

[32] H. Hu, R.G. Larson, Marangoni effect reverses coffee-ring depositions, J. Phys. Chem. B. 110 (2006) 7090-7094. doi:10.1021/jp0609232.

[33] J. Denape, J. Lamon, Sliding friction of ceramics: Mechanical action of the wear debris, J. Mater. Sci. 25 (1990) 3592-3604. doi:10.1007/BF00575394. 